\title{
BMJ Open Post-traumatic stress, anxiety and depression following miscarriage or ectopic pregnancy: a prospective cohort study
}

\author{
Jessica Farren, ${ }^{1}$ Maria Jalmbrant, ${ }^{2}$ Lieveke Ameye, ${ }^{3}$ Karen Joash, ${ }^{1}$ \\ Nicola Mitchell-Jones, ${ }^{4}$ Sophie Tapp, ${ }^{1}$ Dirk Timmerman, ${ }^{3,5}$ Tom Bourne ${ }^{1,3,5}$
}

To cite: Farren J,

Jalmbrant M, Ameye L, et al. Post-traumatic stress, anxiety and depression following miscarriage or ectopic pregnancy: a prospective cohort study. BMJ Open 2016;6: 011864 . doi:10.1136/bmjopen-2016011864

- Prepublication history and additional material is available. To view please visit the journal (http://dx.doi.org/ 10.1136/bmjopen-2016011864).

Received 12 March 2016 Revised 11 August 2016 Accepted 16 August 2016

CrossMark

For numbered affiliations see end of article.

Correspondence to Professor Tom Bourne; t.bourne@imperial.ac.uk

\section{ABSTRACT}

Objectives: This is a pilot study to investigate the type and severity of emotional distress in women after early pregnancy loss (EPL), compared with a control group with ongoing pregnancies. The secondary aim was to assess whether miscarriage or ectopic pregnancy impacted differently on the type and severity of psychological morbidity.

Design: This was a prospective survey study. Consecutive women were recruited between January 2012 and July 2013. We emailed women a link to a survey 1,3 and 9 months after a diagnosis of EPL, and 1 month after the diagnosis of a viable ongoing pregnancy.

Setting: The Early Pregnancy Assessment Unit (EPAU) of a central London teaching hospital.

Participants: We recruited 186 women. 128 had a diagnosis of EPL, and 58 of ongoing pregnancies. 11 withdrew consent, and 11 provided an illegible or invalid email address.

Main outcome measures: Post-traumatic stress disorder (PTSD) was measured using the Posttraumatic Diagnostic Scale (PDS), and anxiety and depression using the Hospital Anxiety and Depression Scale (HADS).

Results: Response rates were 69/114 at 1 month and 44/68 at 3 months in the EPL group, and 20/50 in controls. Psychological morbidity was higher in the EPL group with $28 \%$ meeting the criteria for probable PTSD, $32 \%$ for anxiety and $16 \%$ for depression at 1 month and $38 \%, 20 \%$ and $5 \%$, respectively, at 3 months. In the control group, no women met criteria for PTSD and 10\% met criteria for anxiety and depression. There was little difference in type or severity of distress following ectopic pregnancy or miscarriage.

Conclusions: We have shown a large number of women having experienced a miscarriage or ectopic pregnancy fulfil the diagnostic criteria for probable PTSD. Many suffer from moderate-tosevere anxiety, and a lesser number depression. Psychological morbidity, and in particular PTSD symptoms, persists at least 3 months following pregnancy loss.

\section{Strengths and limitations of this study}

- We have used validated instruments to obtain quantitative data on mental well-being after early pregnancy loss.

- We have included women who have suffered both miscarriages and ectopic pregnancies.

- In addition to anxiety and depression we have tested for the probable presence or absence of post-traumatic stress disorder.

- The main limitation of this study was the dropout rate for responses at 1 month and in our control group.

- While it is a strength that we have included a control group, a potential weakness is that these were women who were attending an early pregnancy unit for assessment.

\section{INTRODUCTION}

Early pregnancy loss (EPL) is common, with miscarriage affecting $25 \%$ of women who have been pregnant by the age of 39, constituting 12-20\% of all pregnancies. ${ }^{1}$ Ectopic pregnancy (defined as the development of a pregnancy outside the uterine cavity, usually within the fallopian tube) is less common, thought to occur in $\sim 1 \%$ of pregnancies. ${ }^{2}$

The psychological consequences associated with an EPL cannot necessarily be extrapolated from our understanding of grief reactions in other contexts. They encompass both bereavement, and an often traumatic (and in some cases life-threatening) personal physical experience. They can pose a potential threat to the dream or expectation to have a family. In contrast to other losses, there are no standardised rituals to manage grief, and there is often no physical manifestation of the loss to mourn. Societal norms may also encourage privacy, which may translate into less support from friends or colleagues. 
Previous studies evaluating the immediate psychological impact of miscarriage have shown a significant proportion of women meet the criteria for depression $(27 \%)$ and anxiety $(28-41 \%) \cdot{ }^{3-5}$ Such symptoms decline over time, to levels equivalent to the non-pregnant population at 1 year. ${ }^{6}$ In general, anxiety is more marked than depression. ${ }^{78}$

Post-traumatic stress disorder (PTSD) may develop in response to experiencing a real or perceived threat of death or injury to oneself or others. In the context of EPL, the threat of serious injury, sight of blood or fetal tissue, or subjective perception of experiencing the death of a baby may be sufficiently traumatic to result in PTSD. In previous studies, women have rated EPL as their worst lifetime exposure to trauma. ${ }^{9}$ There are few data relating to PTSD and miscarriage. In one prospective study involving 113 women, $25 \%$ met the criteria according to the self-report symptom scale for PTSD at 1 month, and $7 \% 4$ months following an EPL. ${ }^{10}$ However, this study included late pregnancy losses, so it is not possible to establish the prevalence of PTSD after a loss in early pregnancy from these results.

There are few published data evaluating psychological morbidity following ectopic pregnancy. One retrospective study has suggested there may be an association between surgically treated ectopic pregnancies and suicide. ${ }^{11}$ Ectopic pregnancy is a potentially lifethreatening condition that is more likely to lead to emergency hospital admission and surgical intervention, where the pregnancy loss is often treated as secondary. Furthermore, ectopic pregnancy is frequently associated with a threat to future fertility. Given this enhanced threat of physical harm in addition to the EPL itself, it might be expected that the psychological consequences are different.

An understanding of the type and frequency of emotional reactions to pregnancy loss is important in order to target appropriate support to those that need it, thereby minimising psychological morbidity and its societal cost. In the current study, the primary aim was to assess the symptoms of PTSD, anxiety and depression in women following EPL compared with a control group of women with ongoing viable pregnancies. The secondary aim was to evaluate whether there was any evidence to suggest different levels of PTSD, anxiety and depression symptomatology in women who had suffered a miscarriage compared with those with an ectopic pregnancy. A final aim was to explore the feasibility and sample size required for a larger study to assess for risk factors, which could be used to target screening or treatment of psychiatric morbidity in this context.

\section{METHODS}

\section{Design}

We recruited women presenting to the Early Pregnancy Assessment Unit (EPAU) at Queen Charlottes and Chelsea hospital, which is a central London university teaching hospital. Women attended the unit because of bleeding and/or pain, or for reassurance or dating. The data represent a pilot to demonstrate the feasibility of, and provide data from which to derive the sample size for, a larger study examining risk factors for the development of psychological morbidity following EPL.

On the days on which the study investigators were available (an average of one-half day per week), consecutive women were approached for recruitment. Initially, we approached all women who had been diagnosed on the basis of a transvaginal ultrasound scan as having an ectopic pregnancy or miscarriage. Women were approached on the day of their diagnosis, or at subsequent follow-up visit. Following a change in the study protocol in December 2012 to enable a control group to be recruited, we approached all women attending the EPAU, including those with a pregnancy of unknown location and ongoing viable pregnancies. Hospital records were checked to confirm the outcome of the pregnancy.

Women were offered management in accordance with local protocols. Those with ongoing viable pregnancies were discharged and requested to book their standard antenatal care. Those with miscarriage (unless complete) were offered the clinically appropriate options out of expectant, medical (with misoprostol administered by the patient, per vaginam, at home) or surgical management (suction evacuation under general anaesthetic). Women with ectopic pregnancies were offered expectant, medical (with a single dose of methotrexate) or surgical (usually laparoscopic salpingectomy) management, according to what was clinically appropriate with respect to their symptoms, the findings on ultrasonography and serum human chorionic gonadotrophin levels.

All women recruited to the study were sent a link to a survey by email 1 month after diagnosis of their EPL, or at first confirmation of viability (defined as visualisation of embryo heart activity). Women with an EPL who completed the 1-month questionnaire were also requested to complete surveys at 3 and 9 months following the diagnosis. These time points were chosen to measure prolonged distress, and also to avoid both the due date of the lost pregnancy (which would usually be after 6-8 months), and the anniversary of the loss, both of which might be expected to exacerbate distress.

Written consent was gained for all participants. In every communication, including a confirmatory email sent 48 hours after recruitment, the participant was reminded that their participation was voluntary, and that they were free to withdraw.

In the 3-month and 9-month follow-up questionnaires, women were asked whether they were trying to conceive, were pregnant or had suffered another loss.

\section{Participants}

Patients were approached if they were above the age of 18 years, were able to give informed consent, could 
speak English sufficiently to consent and respond to questions in the study, and if the gestational age of the pregnancy was $<20$ weeks.

Between 1/1/2012 and 1/7/2013, 198 eligible women were consecutively approached and 186 women were recruited (figure 1). Of those who declined to take part (12 cases), and voluntarily provided a reason, the majority explained that they did not want to be reminded of the event by the questionnaires. One woman explained that she had strong faith, and believed that the loss was 'in God's hands'-and explained that she felt participating and 'questioning' how she felt was against her faith. Eleven women subsequently withdrew their consent by email. None provided a reason for doing so. Eleven women provided illegible or invalid email addresses.

A total of 164 women received questionnaires: 114 had experienced an EPL and 50 had ongoing viable pregnancies (the control group). Clinical information on each participant was collected at enrolment, and updated on completion of follow-up. This included the reason for their initial review, the initial and final diagnosis, and the clinical management. Information relating to the gestational age and type of pregnancy loss and other demographic information about all study recruits are seen in table 1 .

\section{Demographic and background information}

Respondents were asked to provide demographic information including their age, level of education, religion, income, marital status, length of time with current partner, religion, previous mental health problems and ethnic origin. They were then asked whether they had ever had treatment in hospital, or emergency surgery, and to specify the details of this. The participant was then asked whether or not they were still pregnant and they were separated into two streams (control and EPL group) based on their responses.

Both groups were asked to detail any symptoms related to their visit (including the severity of any pain and/or bleeding). They were asked details of their past obstetric history, including any terminations of pregnancy, fertility treatment, the time taken to conceive the index pregnancy and how much the pregnancy was desired. They were asked whether they received clear information from, and were emotionally supported by, healthcare professionals. They were asked how satisfied they were with the healthcare they received, and whether they had received any formal counselling, or would have liked to have been offered it. In addition, the EPL group were asked how worried they were for their own well-being during the loss, how distressed they were and how responsible they felt for the loss.

\section{Measures}

The participants were asked to complete a range of questionnaires presented in the same order, as follows below. For the purposes of this pilot study, we limited our analysis to responses at 1 and 3 months and for questionnaires relating to anxiety, depression and PTSD.

\section{Post-traumatic Diagnostic Scale}

This is a well-validated self-report questionnaire for the presence of post-traumatic stress. ${ }^{12}$ It contains 17 items based on the Diagnostic and Statistical Manual of Mental Disorders (DSM)-IV diagnostic criteria for PTSD and measures probable PTSD diagnosis and symptom severity (a score out of 51, with $>10$ designating at least moderate severity). It has good psychometric properties across a range of populations, with a sensitivity of 0.89 and specificity of 0.75 , and a Cronbach's $\alpha$ coefficient of internal consistency of 0.92 for total symptom severity. ${ }^{13}$ The respondents in the EPL group were asked to rate their symptoms in relation to the pregnancy loss, while the control group was asked to complete the questionnaire in the standard way, in relation to a self-identified exposure to other trauma (if present).

\section{Hospital Anxiety and Depression Scale}

All participants were asked to complete this 14-item (7 questions related to each of anxiety and depression) questionnaire. ${ }^{14}$ Each subscale measures symptom severity (a score out of 21 , with $\geq 11$ indicating moderate and $\geq 16$ severe symptoms). The Hospital Anxiety and Depression Scale (HADS) has good psychometric properties, with Cronbach's $\alpha$ coefficient of internal consistency a mean of 0.83 for anxiety, and 0.82 for depression, and has been used in a range of populations; a review article identified 747 papers using HADS by May 2000. ${ }^{15}$

Other self-report questionnaires completed by the participants but not included in this analysis are the State Trait Anxiety Inventory (STAI), Norbeck Social Support Questionnaire (NSSQ), the Rumination Response Scale (RRS) and Self-compassion Scale-short form (SCS).

The 3-month and 9-month questionnaires did not repeat demographic questions. They asked if there had been any change in relationship status or new medical conditions, as well as whether they had received any counselling and, if so, whether it had been helpful. It then asked whether they had tried to conceive or were pregnant again. Respondents were then directed to HADS, Post-traumatic Diagnostic Scale (PDS), NSSQ RRS and SCS.

Only a minority of participants responded to the 9-month questionnaire (18/68 (26\%) of those emailed). These results have therefore not been analysed for the pilot study, and this highlighted to us the need to make an ethical amendment to allow us to send participants reminder emails to chase responses.

\section{Statistical analysis}

For the purposes of this paper, we have limited ourselves to an analysis of the data for HADS and PDS. To compare differences in binary variables we used the likelihood ratio, $\chi^{2}$ or Fisher's exact test. We considered a $\mathrm{p}$ value $<0.05$ as statistically significant. Ninety-five per 
Figure 1 Flow chart showing number of women invited to participate, reasons for withdrawal and response rates to 1-month and 3-month questionnaires.
Invited to participate $(n=198)$

\section{Declined to participate $(n=12)$}

Study sample $(n=186)$

Active request to withdraw ( $n=11)$

Invalid or illegible email address $(n=11)$

Study sample sent 1 month questionnaire $(n=164)$

Viable, ongoing pregnancies $(n=50)$

Pregnancy losses $(n=114)$

Respondents to 1 month questionnaire $(n=89)$

Viable, ongoing pregnancies ( $n=20$ )

Pregnancy losses $(n=69)$

\section{Inaccurate clinical information $(n=1)$}

Study sample sent 3 month questionnaire

Known pregnancy losses only $(n=68)$

No response $(n=22)$

No study number filled in $(n=2)$

Respondents to 3 month questionnaire $(n=44)$

Table 1 Demographic and background characteristics of the study sample

\begin{tabular}{|c|c|c|c|c|}
\hline & All recruits & Viable pregnancy & Miscarriage & Ectopic \\
\hline Number recruited & 175 & 56 & 95 (including 13 resolved PUL) & 24 \\
\hline Mean age (years) & 33.8 & 34.1 & 33.0 & 34.9 \\
\hline Mean gestation at diagnosis (weeks) & 9.2 & 10.3 & 9.1 & 7.1 \\
\hline IVF pregnancy (\%) & $10 / 175(6)$ & $4 / 56(7)$ & $6 / 95(6)$ & $0 / 24(0)$ \\
\hline First ultrasound inconclusive (\%) & $76 / 175(43)$ & $13 / 56(23)$ & $57 / 95(60)$ & $6 / 24(25)$ \\
\hline \multicolumn{5}{|l|}{ Final management (\%) } \\
\hline None required/expectant & & NA & $35(37)$ & $1(4)$ \\
\hline Medical management ${ }^{\star}$ & & & $15(16)$ & $2(8)$ \\
\hline Surgical management & & & $43 \dagger(45)$ & $21 \neq(88)$ \\
\hline Lost to follow-up & & & $2(2)$ & $0(0)$ \\
\hline
\end{tabular}


cent CIs for rates of psychological morbidity were created using the Wilson method. All statistical analyses were performed using SPSS V.20 and GraphPad Prism V.6.

\section{RESULTS \\ Demographic and background characteristics of the study sample}

The groups (viable pregnancy, miscarriage and ectopic pregnancy) were similar in terms of age at presentation (see table 1). Gestation at presentation was lower in the ectopic pregnancy group. Similar proportions were in vitro fertilisation (IVF) pregnancies in the control and miscarriage group, but no women with an ectopic pregnancy had IVF. Most ectopic pregnancies were diagnosed on the first ultrasound scan $(18 / 24,75 \%)$. Most miscarriages had an initially inconclusive ultrasound scan $(57 / 95,60 \%)$. The majority of ectopic pregnancies $(21 / 24,88 \%)$ were ultimately treated surgically, whereas $50 / 95(53 \%)$ of miscarriages were successfully managed expectantly or with outpatient medical management.

\section{Response rates}

At 1 month, the response rate for women with EPL was $69 / 114(61 \%)$. This includes one woman who, according to our clinical records had been diagnosed with a viable pregnancy, but in her responses indicated that a miscarriage had taken place while abroad. This participant did not receive the subsequent questionnaires. The response rate for the control group was significantly lower: 20/50 (40\%; $\mathrm{p}=0.02)$.

At 3 months, $44 / 68(65 \%)$ of women responded. In addition, there were three completed questionnaires (one at 1 month (who self-reported a loss), and two at 3 months) in which the respondent did not fill in their study number. These were excluded from the analysis.

\section{Respondents}

Online supplementary table S1 details background information on respondents in the viable and EPL groups. Both groups had similar characteristics in terms of past obstetric and psychiatric history.

\section{Levels of post-traumatic stress}

At 1 month, $19 / 69$ (28\% (95\% CI $18 \%$ to $39 \%)$ ) women in the EPL group met the PDS criteria for probable moderate or severe PTSD. In addition, 9/69 (13\%) women met all criteria for probable moderate or severe PTSD except the criterion requiring the symptoms they reported to have been present for more than 4 weeks. At 3 months $17 / 44(39 \%$ (26\% to $53 \%)$ ) met the criteria for probable moderate or severe PTSD. The mean severity score for all respondents with EPL was 15.3 (SD 9.9) at 1 month, and 11.6 (SD 9.5) at 3 months.

We examined the endorsement of separate symptom clusters. Re-experiencing was the most commonly endorsed, followed by avoidance, and then hyperarousal. At 1 month, of all questions asked, the most commonly endorsed statement was-'feeling emotionally upset when you were reminded of the loss of your pregnancy' (40/69 (58\%) women describing this happening at least 2-4 times per week), followed by-'having upsetting thoughts or images about the loss of your pregnancy that came into your head when you did not want them' (30/69 (43\%) women describing this at least 2-4 times per week). At 3 months, re-experiencing remained the most commonly endorsed symptom cluster. Endorsement of avoidance symptoms had declined $(50 / 69(72 \%(61 \%$ to $82 \%))$ to $22 / 44(50 \%$ $(36 \%$ to $64 \%)), \mathrm{p}=0.07)$, and become the least common. The reported impact of the endorsed symptoms on areas of respondents' lives is detailed in table 2.

The dropout rate between 1 and 3 months was slightly (non-significantly) higher for those with probable moderate-to-severe PTSD at 1 month (8/19 (42\%)), than for those with no or mild PTSD (16/49 (33\%)).

In the control group, no women met the criteria for a diagnosis of probable PTSD for self-reported traumatic life events (not specifically probing for previous EPLs).

\section{Levels of anxiety and depression}

At 1 month, 22/69 (32\% (22\% to 44\%)) of women in the EPL group met criteria for moderate-to-severe anxiety, compared with $2 / 20(10 \%$ (3\% to $30 \%))$ in the control group ( $\mathrm{p}=0.04$; table 3 and figure 2$)$. This declined over time in the EPL group to 9/44 (20\% $(11 \%$ to $35 \%))$ at 3 months. The prevalence of moderate and severe depression symptoms was similar in both groups for all time points: 11/69 (16\% (9\% to 26\%)) for women with an EPL compared with 2/20 (10\% (3\% to $30 \%)$ ) for controls, dropping to lower than the control group (2/44; $5 \%(1 \%$ to $15 \%))$ at 3 months.

The mean severity score at 1 month in the EPL group was 7.8 for anxiety (SD 4.8) and 5.7 (SD 4.4) for depression, while the control group had a mean severity score of 6.6 (SD 3.0) for anxiety and 3.9 (SD 4.2) for depression. At 3 months the mean severity score in the EPL group for anxiety was 6.8 (SD 4.2), and 4.2 for depression (SD 3.6).

The dropout rate between 1 and 3 months was higher in women with moderate-to-severe anxiety and depression compared with those with no or mild anxiety or depression, although these results did not reach significance $(11 / 22(50 \%)$ dropout for those with moderate/ severe anxiety vs $13 / 46$ (28\%) with none/mild anxiety, $6 / 11(55 \%)$ vs $18 / 57$ (32\%) respectively for depression).

\section{Ectopic pregnancy compared with miscarriage}

Table 3 compares the psychological distress following ectopic pregnancy and miscarriage. When comparing the ectopic pregnancy and the miscarriage groups, 6 of the 16 women who had experienced an ectopic pregnancy met the criteria for probable PTSD (38\% (18\% to $61 \%)$ ), compared with $13 / 53$ (25\% (15\% to $38 \%)$ ) in the miscarriage group. Four of the 16 (25\% (10\% to $50 \%)$ ) met the criteria for moderate/severe anxiety 
Table 2 Proportion of respondents meeting criteria for post-traumatic stress disorder at 1 and 3 months (subdivided according to severity, including and excluding the duration criterion), individual symptom clusters within PDS endorsed, and areas of life symptoms reported to have impacted on

\begin{tabular}{|c|c|c|c|}
\hline & $\begin{array}{l}1 \text { month } \\
\text { Including duration } \\
\text { criterion }^{*}\end{array}$ & $\begin{array}{l}\text { Excluding duration } \\
\text { criterion* }^{\star}\end{array}$ & 3 months \\
\hline $\begin{array}{l}\text { Total proportion meeting PDS } \\
\text { criteria }(\%(95 \% \mathrm{CI}))\end{array}$ & $22 / 69(32 \%[22 \%$ to $44 \%])$ & $31 / 69(45 \%[34 \%$ to $57 \%])$ & $19 / 44(43 \%[30 \%$ to $58 \%])$ \\
\hline Mild & $3 / 69(4 \%[1 \%$ to $12 \%])$ & $3 / 69(4 \%[1 \%$ to $12 \%])$ & $2 / 44(5 \%[1 \%$ to $15 \%])$ \\
\hline Moderate & $6 / 69(9 \%[4 \%$ to $18 \%])$ & $11 / 69(16 \%[9 \%$ to $26 \%])$ & $9 / 44(20 \%[11 \%$ to $35 \%])$ \\
\hline Moderate to severe & $10 / 69(14 \%[8 \%$ to $25 \%])$ & $14 / 69(20 \%[12 \%$ to $31 \%])$ & $7 / 44(16 \%[8 \%$ to $29 \%])$ \\
\hline Severe & $3 / 69(4 \%[1 \%$ to $12 \%])$ & $3 / 69(4 \%[1 \%$ to $12 \%])$ & $1 / 44(2 \%[0.4 \%$ to $12 \%])$ \\
\hline \multicolumn{4}{|c|}{ Symptom clusters endorsed $(\%, 95 \% \mathrm{Cl})$} \\
\hline Re-experiencing & $64 / 69(93 \%$ [84\% to $97 \%])$ & & $39 / 44(89 \%[76 \%$ to $95 \%])$ \\
\hline Avoidance & $50 / 69(72 \%[61 \%$ to $82 \%])$ & & $24 / 44(55 \%[40 \%$ to $68 \%])$ \\
\hline Hyperarousal & $39 / 69(57 \%$ [45\% to $68 \%])$ & & $27 / 44(61 \%[47 \%$ to $74 \%])$ \\
\hline \multicolumn{4}{|c|}{ Areas of life symptoms impacted on $(\%, 95 \% \mathrm{Cl})$} \\
\hline Work & $36 / 69(52 \%$ [41\% to $64 \%])$ & & $13 / 44(30 \%[18 \%$ to $44 \%])$ \\
\hline Household chores & $22 / 69(32 \%[22 \%$ to $44 \%])$ & & $9 / 44(20 \%[11 \%$ to $35 \%])$ \\
\hline Relationships with family & $28 / 69(41 \%[30 \%$ to $52 \%])$ & & $16 / 44(36 \%[24 \%$ to $51 \%])$ \\
\hline Relationships with friends & $34 / 69(49 \%$ [38\% to $61 \%])$ & & $17 / 44(39 \%[26 \%$ to $53 \%])$ \\
\hline Fun and leisure activities & $38 / 69(55 \%[43 \%$ to $66 \%])$ & & $21 / 44(48 \%[34 \%$ to $62 \%])$ \\
\hline Sex life & $48 / 69(70 \%[58 \%$ to $79 \%])$ & & $21 / 44(48 \%[34 \%$ to $62 \%])$ \\
\hline General satisfaction with life & $48 / 69(70 \%[58 \%$ to $79 \%])$ & & $26 / 44(59 \%[44 \%$ to $72 \%])$ \\
\hline Overall level of functioning & $35 / 69(51 \%[39 \%$ to $62 \%])$ & & $20 / 44(45 \%[32 \%$ to $60 \%])$ \\
\hline
\end{tabular}

symptoms, compared with $18 / 53(34 \%(23 \%$ to $47 \%))$ in the miscarriage group. Two of the $16(13 \%$ (4\% to $36 \%)$ ) met criteria for moderate/severe depression symptoms, compared with $9 / 53(17 \%(9 \%$ to $29 \%))$ in the miscarriage group. None of these differences reached statistical significance.

\section{Comparison according to pregnancy status at 3 months}

At 3 months, 22/44 (50\%) of respondents reported trying to conceive but not yet being pregnant. Nine of the $22(41 \%$ (23\% to $61 \%)$ ) who were trying to conceive met criteria for probable PTSD, compared with 2/6 $(33 \%(10 \%$ to $70 \%))$ who were pregnant again, and 6/ $16(38 \%(18 \%$ to $61 \%))$ who had not tried to conceive. Five of the $22(23 \%(10 \%$ to $43 \%))$ of those who were trying to conceive met the criteria for moderate/severe anxiety, compared with none $(0 / 6)$ of the group who were pregnant again, and 4/16 (25\% (10\% to 50\%)) in the group who had not tried to conceive. For depression, $1 / 22(5 \%$ ( $1 \%$ to $22 \%))$ who were trying to conceive, $1 / 6(17 \%$ (3\% to $56 \%)$ ) who were pregnant again and none $(0 / 16)$ of the group who had not tried to conceive met criteria.

\section{DISCUSSION}

We have shown in our cohort of women attending an inner London EPAU that $39 \%$ of participants 3 months after suffering an EPL meet the criteria for probable moderate-to-severe PTSD. Furthermore, 20\% meet the criteria for moderate-to-severe anxiety compared with $10 \%$ in our control population. Levels of anxiety and depression dropped between 1 and 3 months, but the symptoms associated with PTSD persisted. We also found that women with ectopic pregnancy suffer from significant levels of emotional distress, with slightly higher levels of PTSD symptoms compared with women who experience a miscarriage, but lower levels of anxiety and depression.

This pilot study has demonstrated the feasibility of a larger scale study into the psychological morbidity associated with EPL. Women were generally happy to be approached and take part. All women who got as far as entering their study number at the start of the online questionnaire completed all questions, reassuring us that the required time commitment was appropriate. The dropout rate has implications for future study size, and has resulted in us submitting an amendment to allow up to two reminder emails in an effort to improve this.

One of the strengths of this study is that women were consecutively recruited rather than self-referring themselves for involvement, with a high proportion $(94 \%)$ of those approached providing consent to take part. Knowledge of their clinical data and outcomes enabled us to send the questionnaires at appropriate set time points after diagnosis. We also involved a relatively large 
๙ัญ กิ กิ mำ

$+2$

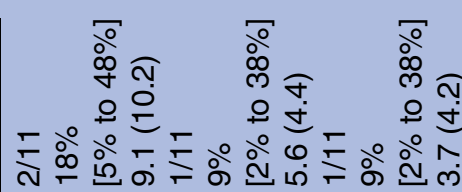
(2) लें ก)

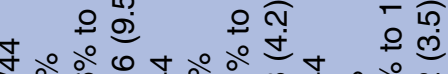
$\frac{\sqrt{2}}{\frac{8}{\circ}}$ $\stackrel{\frac{9}{2}}{\frac{8}{2}}$ ๖ำ ติ 우 ल्

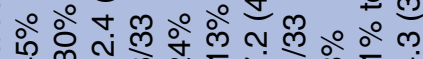

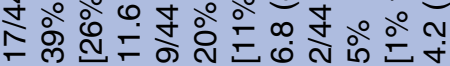
$\frac{\circ}{\circ}$ 萡 ั̊ำ $\circ$ ต

ஓ্’ ஸे ลุ.<smiles>[Li][13IH]</smiles>

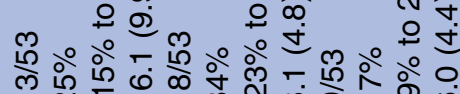

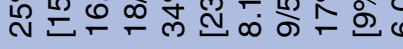

\section{ळे}

¿̊ำ

¿̊ำ

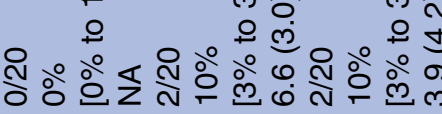

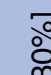

ठ̊ํํ

○

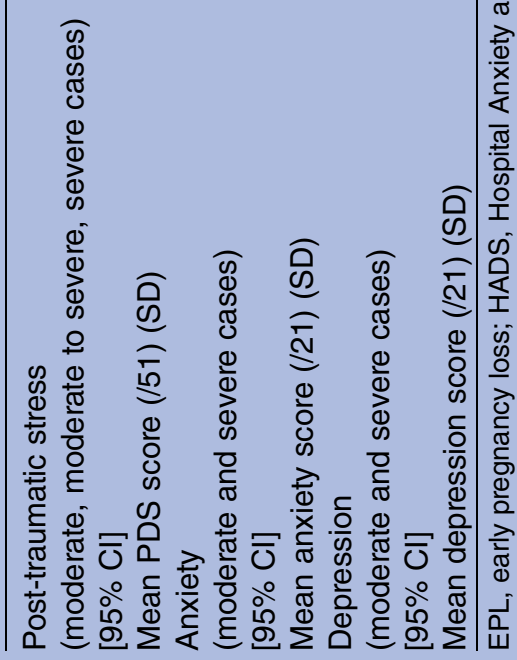

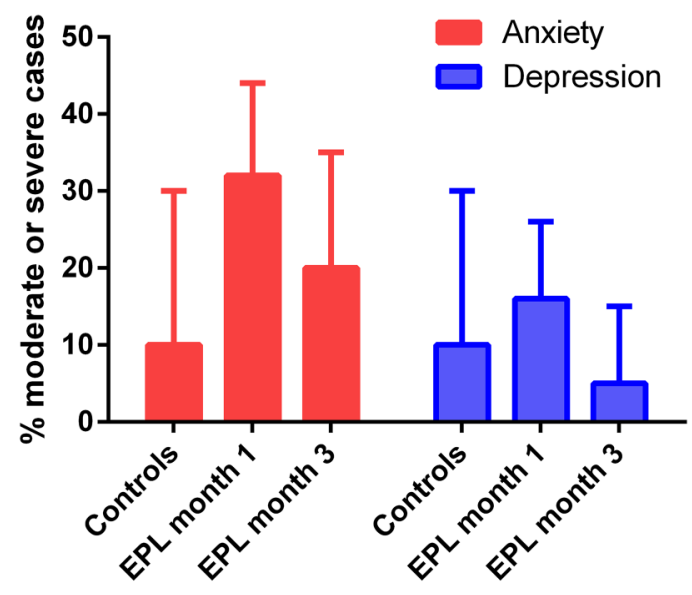

Figure 2 Bar chart illustrating proportion of women meeting criteria for moderate or severe anxiety/depression, according to HADS, with $95 \%$ confidence limits. EPL, early pregnancy loss; HADS, Hospital Anxiety and Depression Scale.

number of women compared with other studies of this type. A further strength is in the inclusion of women with ectopic pregnancies, a group largely unreported in the literature to date.

The primary weakness of the study is in the significant dropout rate, including both a failure to respond to the initial questionnaire, and the dropout of initial responders to subsequent questionnaires. In line with our protocol, it was not possible to find out the reasons for this. It does raise the concern of a potential ascertainment bias artificially inflating rates of pathology. However, while it is possible that women with a more significant emotional response to EPL were more likely to respond, it is also likely that women with the greatest trauma avoid engagement with a process that makes them confront the event. The observed selective dropout of those with higher PTSD, anxiety and depression levels perhaps supports the view that there may have been a group of women who did not want to be reminded of their feelings about their EPL. A further weakness is the possibility of an unintended, moderating therapeutic effect from taking part in the study-a hypothesis supported by results from Neugebauer et $a l \mathrm{~s}^{16}$ study. Finally, we recognise that this study relies on the use of self-report screening questionnaires, whereas confirmed diagnosis of these disorders requires more extensive interviews with appropriately trained personnel.

Respondents in our control group who did not suffer an EPL in the index pregnancy had a high rate of previous miscarriage $(45 \%)$. This can be explained by the fact that in the UK, attendance for medical review in early pregnancy is often for reassurance in women with a previous history of EPL. It also seems likely that women with a past pregnancy loss would be more likely to invest time in the questionnaire. The result is that, while our control group is a useful comparator, we would probably be underestimating overall levels of 
distress related to EPL if we were to rely on it as a normative baseline. Our control group is also smaller than the EPL group, as a result of the recruitment methodology described, which has implications for the power of the EPL versus control group calculations.

We were surprised by the prevalence of PTSD symptoms in our study. Even in women not meeting the full criteria for PTSD, there was a significant endorsement of all symptom clusters by the majority of participants, along with impairment across social and occupational domains, and in general satisfaction. This prevalence of probable PTSD is higher than previously reported. ${ }^{10}$ However, in the paper by Engelhard and colleagues, the participants were not limited to women with losses in early pregnancy. Furthermore, the PDS was scored differently in our paper. We scored according to Foa's original recommendation-where a symptom was considered endorsed if it occurs 'once a week or less/once in a while'-whereas Engelhard required a symptom to occur '2-4 times a week/half the time'.

It is noteworthy that there is an apparent increase in probable PTSD between month 1 (28\% of respondents) and month $3(38 \%)$. This reflects the timing of the questionnaires, which were sent out 1 month after the EPL. Accordingly women may have endorsed the symptom clusters that constitute a diagnosis of PTSD, but would not at that stage have fulfilled the requirement for the symptoms to have been present for at least 1 month. We considered classifying these women as having an acute stress disorder; however, strictly speaking the PDS is not validated for this purpose. In any event the number of women who endorse the symptoms clusters for PTSD other than the duration criterion at 1 month is $41 \%$ of initial respondents, suggesting that there is no appreciable fall off in PTSD symptoms between months 1 and 3 .

The observation that such a significant proportion of women suffer from symptoms of PTSD after losing a pregnancy, and that this is more common than both anxiety and depression, is potentially important. In other contexts, we know that untreated PTSD has a significant impact on quality of life, relationships, ability to work, suicide risk and physical health. ${ }^{17-19}$ PTSD also has implications for future pregnancies, including poor health behaviour, reduced gestational length and issues with infant bonding. ${ }^{20-22}$ The treatment for PTSD is specific and relies on trauma-focused cognitive-behavioural therapy or eye movement desensitisation and reprocessing. ${ }^{23}$ It is perhaps not surprising therefore that the outcome of a broad-brush approach of offering 'counselling' (in variable formats) to all women following miscarriage has been disappointing. ${ }^{24}$ Given the serious impact of untreated PTSD, screening women for the disorder following EPL may be a more effective use of resources. Conversely, we have shown that there are a number of women who do not suffer significant morbidity. These may not require any intervention following their loss.
There are some issues to be considered with respect to the diagnosis of PTSD using the PDS. First, the diagnostic criteria for PTSD changed slightly during the trial. In the 2013 DSM-V, the requirement for the trauma to result in fear, helplessness or horror was removed. ${ }^{18}$ All of our patients with the other features of PTSD met this criterion; thus, its removal would not change our results. In addition, a fourth symptom cluster relating to negative alterations in mood was included. The PDS does not contain questions pertaining to this cluster, and there are currently no validated screening questionnaires relating to it. In any event, although diagnostic thresholds are important to define the nature of any pathology, this change does not detract from the frequency or severity of distress we have observed. Third, there may be debate about miscarriage or ectopic pregnancy fulfilling criteria for a 'traumatic event' according to DSM-V ${ }^{18}$ which requires either participation in a life-threatening event, including a catastrophic medical event, or observing the death or serious injury to others. Clearly, some EPLs do result in sudden, life-threatening haemorrhage or emergency surgery and may thus be classified as a catastrophic medical event. The interpretation of whether an EPL fulfils the criteria of vicarious trauma by observing death or serious injury is subjective. Some women and couples start relating to their embryo as a child as soon as they have received a positive pregnancy test, and for them losing that embryo and perhaps observing the embryo or fetus being passed during the miscarriage may be equated with the catastrophic death of a child.

Future research should be aimed at assessing the risk factors for PTSD. Based on this pilot study, we have estimated that a total of 440 women with losses would need to complete part 1 of the study to demonstrate a $20 \%$ difference in prevalence between women with assisted reproduction and those without (chosen as the least common potential risk factor of interest; power 0.80 and $\alpha 0.05)$. Accounting for rates of declined participation, withdrawal and non-response, 811 women with EPL should be recruited.

Our findings are relevant to healthcare professionals who deal with EPL. Exposure to EPL on a daily basis may lead clinicians to normalise the experience and overlook the possible profound psychological sequelae. The data presented are in the context of a pilot study; however, if our findings are supported by further large prospective studies, we believe that consideration should be given to screening all women who have suffered an EPL for PTSD. There is also a need to assess how to predict those women who are most at risk of serious psychological morbidity, in order to better direct limited resources, and to facilitate early intervention and appropriate treatment.

Author affiliations

${ }^{1}$ Tommy's National Centre for Miscarriage Research, Queen Charlottes and Chelsea Hospital, Imperial College, London, UK 
${ }^{2}$ South London and Maudsley NHS Foundation Trust, London, UK

${ }^{3}$ Department of Development and Regeneration, KU Leuven, Leuven, Belgium

${ }^{4}$ Chelsea and Westminster NHS Trust, London, UK

${ }^{5}$ Department of Obstetrics and Gynaecology, University Hospitals Leuven,

Leuven, Belgium

Twitter Follow Tom Bourne at @proftombourne

Acknowledgements The research team would like to thank the women who generously donated their time to participate, as well as all the staff in the EPU for their support with this study.

Contributors TB, MJ and KJ devised the original study protocol. JF recruited patients for the study. JF and LA were responsible for statistical analysis of the results. TB, JF and MJ wrote the first draft of the manuscript that was then critically reviewed and revised by the other co-authors. DT, NM-J and ST commented on the drafts of the paper. All authors approved the final version of the manuscript for submission. All authors had full access to all of the data (including statistical reports and tables) in the study and can take responsibility for the integrity of the data and the accuracy of the data analysis. TB is the guarantor, and affirms that the manuscript is an honest, accurate and transparent account of the study being reported; and that any discrepancies from the study as planned have been explained.

Funding JF was supported by Imperial College Healthcare Charity grant number 141517. TB is supported by the National Institute for Health Research (NIHR) Biomedical Research Centre based at Imperial College Healthcare NHS Trust and Imperial College London. The early pregnancy unit at Queen Charlottes and Chelsea Hospital is supported by the Tommy's charity. DT is Senior Clinical Investigator of FWO (Research Foundation-Flanders).

Disclaimer The views expressed are those of the author(s) and not necessarily those of the NHS, the NIHR or the Department of Health.

Competing interests None declared.

Ethics approval This was a prospective cohort study, with ethical approval of the study protocol granted by the NRES committee of South-West Exeter, reference 11/SW/0052.

Provenance and peer review Not commissioned; externally peer reviewed.

Data sharing statement No additional data are available.

Open Access This is an Open Access article distributed in accordance with the Creative Commons Attribution Non Commercial (CC BY-NC 4.0) license, which permits others to distribute, remix, adapt, build upon this work noncommercially, and license their derivative works on different terms, provided the original work is properly cited and the use is non-commercial. See: http:// creativecommons.org/licenses/by-nc/4.0/

\section{REFERENCES}

1. Blohm F, Fridén B, Milsom I. A prospective longitudinal population-based study of clinical miscarriage in an urban Swedish population. BJOG 2008;115:176-82. discussion 83.

2. NICE. Ectopic pregnancy and miscarriage: diagnosis and initial management. Clinical guideline (CG154). Published date: December 2012. https://www.nice.org.uk/guidance/cg154 last accessed 10th October 2016.
3. Lok IH, Yip AS, Lee DT, et al. A 1-year longitudinal study of psychological morbidity after miscarriage. Fertil Steril 2010;93:1966-75.

4. Cumming GP, Klein S, Bolsover D, et al. The emotional burden of miscarriage for women and their partners: trajectories of anxiety and depression over 13 months. BJOG 2007;114:1138-45.

5. Prettyman RJ, Cordle CJ, Cook GD. A three-month follow-up of psychological morbidity after early miscarriage. Br J Med Psychol 1993;66:363-72.

6. Janssen HJ, Cuisinier MC, Hoogduin KA, et al. Controlled prospective study on the mental health of women following pregnancy loss. Am J Psychiatry 1996;153:226-30.

7. Thapar AK, Thapar A. Psychological sequelae of miscarriage: a controlled study using the general health questionnaire and the hospital anxiety and depression scale. Br J Gen Pract 1992;42:94-6.

8. Nikcevic AV, Tunkel SA, Nicolaides KH. Psychological outcomes following missed abortions and provision of follow-up care. Ultrasound Obstet Gynecol 1998;11:123-8.

9. Hamama L, Rauch SA, Sperlich M, et al. Previous experience of spontaneous or elective abortion and risk for posttraumatic stress and depression during subsequent pregnancy. Depress Anxiety 2010;27:699-707.

10. Engelhard IM, van den Hout MA, Arntz A. Posttraumatic stress disorder after pregnancy loss. Gen Hosp Psychiatry 2001;23:62-6.

11. Farhi J, Ben-Rafael Z, Dicker D. Suicide after ectopic pregnancy. N Engl J Med 1994;330:714.

12. Foa EB, Riggs DS, Dancu CV, et al. Reliability and validity of a brief instrument for assessing post-traumatic stress disorder. J Trauma Stress 1993;6:459-73.

13. Foa E, Cashman L, Jaycox L, et al. The validation of a self-report measure of posttraumatic stress disorder: the Posttraumatic Diagnostic Scale. Psychological Assessment 1997;9:445-51.

14. Zigmond AS, Snaith RP. The hospital anxiety and depression scale. Acta Psychiatr Scand 1983;67:361-70.

15. Bjelland I, Dahl AA, Haug TT, et al. The validity of the Hospital Anxiety and Depression Scale. An updated literature review. $J$ Psychosom Res 2002;52:69-77.

16. Neugebauer R, Kline J, O'Connor P, et al. Depressive symptoms in women in the six months after miscarriage. Am J Obstet Gynecol 1992;166:104-9.

17. Kessler RC. Posttraumatic stress disorder: the burden to the individual and to society. J Clin Psychiatry 2000;61(Suppl 5):4-12; discussion 13-4.

18. American Psychiatric A, American Psychiatric Association DSMTF. Diagnostic and statistical manual of mental disorders: DSM-5. London: American Psychiatric Association, 2013.

19. Rapaport MH, Clary C, Fayyad R, et al. Quality-of-life impairment in depressive and anxiety disorders. Am J Psychiatry 2005;162:1171-8.

20. Rogal SS, Poschman K, Belanger K, et al. Effects of posttraumatic stress disorder on pregnancy outcomes. J Affect Disord 2007;102:137-43.

21. Dunkel Schetter C, Tanner L. Anxiety, depression and stress in pregnancy: implications for mothers, children, research, and practice. Curr Opin Psychiatry 2012;25:141-8.

22. Klier CM, Geller PA, Ritsher JB. Affective disorders in the aftermath of miscarriage: a comprehensive review. Arch Womens Ment Health 2002;5:129-49.

23. NICE. Post-traumatic stress disorder management. Clinical guideline (CG26). Published date: March 2006. https://www.nice.org.uk/ guidance/cg26 last accessed 10th October 2016

24. Murphy FA, Lipp A, Powles DL. Follow-up for improving psychological well being for women after a miscarriage. Cochrane Database Syst Rev 2012;(3):Cd008679. 